\title{
How the Taiwanese Do China Studies: Applications of Text Mining
}

\author{
Hsuan-Lei Shao ${ }^{1}$, Sieh-Chuen Huang ${ }^{2}$, Yun-Cheng Tsai ${ }^{3}$ \\ ${ }^{1}$ Department of East Asian Studies, National Taiwan Normal University \\ ${ }^{2}$ College of Law, National Taiwan University \\ ${ }^{3}$ Center for General Education, National Taiwan University \\ Corresponding author: Yun-Cheng Tsai, pecutsai@ntu.edu.tw
}

\begin{abstract}
With the rapid evolution of cross-strait situation, "Mainland China" as a subject of social science study has evoked the voice of "Rethinking China Study" among intelligentsia recently. This essay tried to apply an automatic content analysis tool (CATAR) to the journal "Mainland China Studies" (1998-2015) in order to observe the research trends based on the clustering of text from the title and abstract of each paper in the journal. The results showed that the 473 articles published by the journal were clustered into seven salient topics. From the publication number of each topic over time (including "volume of publications", "percentage of publications"), there are two major topics of this journal while other topics varied over time widely. The contribution of this study includes:

1. We could group each "independent" study into a meaningful topic, as a small scale experiment verified that this topic clustering is feasible.

2. This essay reveals the salient research topics and their trends for the Taiwan journal "Mainland China Studies".

3. Various topical keywords were identified, providing easy access to the past study.

4. The yearly trends of the identified topics could be viewed as signature of future research directions.
\end{abstract}

\section{Keywords}

Rethinking China Study; Mainland China Studies; Chinese Communist Party; Structural Topic Model; Text Mining; Support vector machine; Support Distribution Machine; Latent Dirichlet Allocation

\section{INTRODUCTION}

Nowadays, digital method is penetrating into other field broadly. Even in the fields that we think those are not "digital things", such as philosophy, literature and so on. It is called digital humanities $(\mathrm{DH})$. With regard to the theory of digital humanities, there has been considerable discussion nowadays. Besides the applications on each specific fields, DH researchers have gradually been willing to accept the "text structure can be regarded as a numerical data structure" (see Grimmer and Stewart [2013]).

Then use data science methods to calculate documents. It is usually acceptable that "computers can reduce the labour of researchers and open up new insights for them" at least. Therefore, method of text mining is to assist researchers rather than replace them in theoretically. Although there are different scale on how far can humanities phenomenon DH by data science method, Grimmer's viewpoint is still the maximum consensus. Recent researcher has included extending the use of databases and visual plug-ins to study classical Chinese books (see Sturgeon [2017]) or the social networks of a celebrity (see Finegold et al. [2016]) and to capture article 
conversations onto novels (see Muzny et al. [2017]). They are all admit the principle of "text as data" basically.

Much of our research aim to understand which ideas Taiwanese researchers have been eager to know "who studies China on what, when, and how" (as the classical political question: "Who Gets What When and How", (see Lasswell [1936]). To that end, we use text mining technology to analyze published research articles according to their co-occurring words. This will help those who are interested in to have a whole picture in their field. The research described below is about the field of "China Studies in Taiwan" (CSIT). This field is a natural subject for text mining not only the subject is familiar to the authors that we could have sufficient domain knowledge to judge the machine learning models, but also it is undeniably the most important and dynamic influence on Taiwan for now and the near future. As the relationship between mainland China and Taiwan has gradually shifted, the academic circle in Taiwanese has had a growing tendency to put more emphasis on China Studies as it reflects on recent years. About the preliminary research, we have conducted is described below, followed by directions for further research. The contributions of this line of research include the following:

1. These studies reveal the salient research topics and trends in "Mainland China Studies", an academic journal edited by National Chengchi University. It is a typical journal in the China Studies field.

2. Various topical keywords were identified, providing future researchers easy access to the most important information published in Mainland China Studies, without having to read or even skim every individual article.

3. The yearly trends of the identified topics can provide valuable insights to guide future research directions.

4. More broadly, categorizing and grouping independent studies according to meaningful topics can provide an objective birds-eye view of the entire field of CSIT. The main propose of this paper is how data-science method can work/help in a specific academic area. In this manuscript, we also do it in "short text in Chinese". Our target in this stage is to connect "DH (China-studies)" and "DM (we use a digital tool)". This is the spirit of "CS+X", which encourage we use some non-traditional method to our own domain knowledge field.

\section{PRELIMINARIES}

Mainland China is a popular subject of social science research. It has gradually gained significance in Taiwanese academic circle due to its special historical, political and economic relations throughout the world. The political status and social environment of the mainland have changed tremendously since it began its "Reform-and-Opening-Up" policies in the late 1970s, making it a trendy topic around the globe. When it comes to the field of CSIT, Taiwanese scholars offer unique strengths. For one thing, it can be easier for Taiwanese scholars with a solid foundation in Western social science theories to perceive the development and the transformation of China's politics and society from a more objective angle than for many scholars from mainland China who do not rely on the same foundation. In addition, scholars from Taiwan have innate linguistic advantages compared with most Western scholars because the official language in both mainland China and Taiwan is Chinese. As a result, Taiwanese scholars have played an important role in this field while the relationship between Mainland China has got more important weight worldwide in recent years. In the 1990s, social scientists of CSIT began paying more attention to the integration of various methods and approaches within academia. During and since this decade, there has been a radical shift in the approach to CSIT. Such development 
includes, among other things, historical research and paradigm research. The first approach, historical research, marks the characteristics of each period of CSIT. Historical Speaking, CSIT could divide into three periods: Firstly, the banditry studies (before 1970's) have taken Chinese Communist Party (CCP) as an enemy, and focusing on military and war. Secondly, the CCPcentered studies (1970's to 1990's) have focused on factions' struggle in CCP, as Kremlinology in the US. Thirdly, we call it the Mainland China studies period (after 1990's) which is using modern social scientifical method to study.

Nowadays, CSIT has evolved from a focus on combating Communism to interest in business, social, and cultural issues; that is, it has largely shifted from hard politics to soft politics. The social scientifical research conducted through the lens of various disciplines (see Bao and $\mathrm{Wu}$ [2011], Si-Yin Ho and Geng [2005]). In consultation with scholars from different disciplines, they examine China through a variety of theoretical models, including through the theory of integration, the divided nation model, the negotiation model, economic and trade policy, psychological analysis, strategic triangular theory, international system theory, safety code theory and so on. One of the purposes of these books is to provide examples of different research methods in CSIT. But a drawback of paradigm research is that it often presents a static picture, rather than revealing changes in China and in CSIT over time. Objectively, our research falls somewhere between and touches upon both of these approaches. In recent, there are many social science research on text mining or machine learning. For example, Researchers used structural topic model (STM) to help human-hand encoding more effectively (see Roberts et al. [2014]). Another paper showed the classifier machine in the political context (see Bjorn Burscher and Vreese [2015]).

On the hand, algorithms become more complex and more accurate. There are researchers who used support vector machine (SVM) and Support Distribution Machine (SDM), and Latent Dirichlet Allocation (LDA) (see Benedikt Boecking and Schneider [2015]). On the other hand, data scare is growing up as well, Wilkerson and Casas did a good exercise in counting big data (see Wilkerson and Casas [2017]).

\section{METHODOLOGIES}

In theory, most of the DH research papers have not claimed that they have "a theoretical" breakthrough or are trying to establish a "new theory of DH". The above essays are representative of the three trends in the development of DH today: Firstly, to use of data and math-tools to prove existing theories. Such as who visualize six degrees of social network theory (see Muzny et al. [2017]). Secondly, to give proof of empirical data academic arguments that can only remain in the concepts in the past, such as who proves the relationship between the "Confucianism, Daoism, Legalism" among Chinese classics (see Sturgeon [2017]).

This question could only be explained by the researchers' "intuition" before. Third, the invention of new technologies, such as who can achieve higher efficiency and draw on "dialogue", which focuses on technology (see Muzny et al. [2017]). Therefore, with the development of DH research technology and interaction with the original humanities and communities. It can be speculated that there should be three stages of "diversion of technology and theory", "dialogue of technology and theory" and "fusion of technology and theory". Because this research object belongs to the literature interpretation in the traditional research field, and it is still a developing DH research environment which this author is located. Therefore, this article expects its own position from "diversion of technology and theory" to "dialogue of technology and theory", which is also spirit "CS+X". 
We apply a "topic model" methodology, and use Content Analysis Toolkit for Academic Research (CATAR) (see Tseng et al. [2009], Tseng [2010]), which begins with a few basic assumptions:

1. Every article (Document A, Document B, etc. in the figure below) is made up of its smallest meaningful units, words. This does not necessarily mean individual Chinese characters.

2. Looking at both which words co-occur across articles and the frequency of such cooccurrence, we can derive a numeric term that describes the level of similarity in material covered between articles.

3. These numeric terms allow us to compare levels of similarity with greater precision and objectivity than simply reading every article personally.

Documents which are more similar, will be clustered together easier. We can pull out the "cowords" (words co-occurring in multiple articles) in multiple levels. These "co-words" tell us the most important keywords in each cluster of articles. We call these most important co-words "topics", and we use them to build "topic models". The more samples included in the analysis, the more levels the topic model will contain.

Our preliminary research applied this methodology to all of the articles and essays published in the Taiwanese journal titled Mainland China Studies from 1998 to 2015, which were first collected in a database. Then, We constructed topic models using the titles and summaries of each piece. This helped identify the most popular research topics and their keywords, in order to more closely examine the trends in each research topic.

\section{EXPERIMENTAL RESULTS}

The results highlight seven main themes in the 473 pieces published by Mainland China Studies between 1998 and 2015, each theme with its own keywords. Examining the distribution of articles on each topic over time (including both numbers and percentages of articles), we identified two major and enduring topics of the journal (economics/trade and the CCP), in addition to the many other topics that varied over time.

Our method outputs identified three major clusters. The keywords are associated with those clusters. We might characterize them as clusters around economic or social issues, political systems, and political thought. But Figure 1 shows that we look more closely at the keywords and relying on our domain knowledge in the field of CSIT, we further divided the three clusters into seven smaller clusters. These seemed to reflect the distinct themes in each cluster (the first large cluster splitting into three smaller ones, and the two other large clusters each splitting into two smaller ones), as seen below. Certainly, other researchers might choose to divide the machine's outputted clusters differently or not at all.

Using that information, Table 1 shows a time series of common themes and the number of articles Mainland China Studies published on them from 1998 through 2015. Figure 2 shows that the same information can also be visualized by frequency. Figure 3 shows that the same information can also be visualized by percentage.

Clearly, the primary theme is "Economics and Trade" which has relatively steadily accounted for over a third of all published articles. The keywords for this cluster are "economy and trade, negotiation, cross-strait, enterprise, system". It therefore seems likely that the most frequently researched topic for Taiwanese scholars publishing in Mainland China Studies since 1998 relates to doing business with mainland China. The next biggest cluster is the CCP. Its keywords 
are "politics, leadership, decision-making, movement, party and state". This cluster is about political leaders, major political events, and the political party system of the CCP. We can also see that the cluster for "Ideology", never wildly popular, is decreasing in research popularity over time, which is consistent with our experience as a China Studies scholar.

These and other clusters can of course be examined in more granular detail by reading the actual articles within them. We leave that joy of individual discovery to the readers.

\section{CONCLUSION}

Our research proposes the following contributions for the CSIT, among others:

1. The text mining tool could help DHer to know their field in bird eye.

2. Our research pointed different stream of CSIT specifically, such as sociology, economics, or other scholarly topics.

3. In the future, the tool can be applied to study non-academic writing, such as political opinions in newspapers, internet comments on Facebook or Twitter, or other sources of information.

With regards of research limitation, the goal of this paper is to demonstrate the effect of using text mining to study Chinese materials on the one hand; on the other hand, it is also hope to increase the interest of the international academic community in the application of Chinese processing techniques.

Therefore, at this stage, more familiar algorithms are used, such as using simple frequency instead of TF-IDF for basic values, and Jaccard distance for similarity, not cosine distance or Euclidean distance. The comparison of these models can continue to develop in the future. We also agree that "all models are wrong, but some are useful" (see Grimmer and Stewart [2013]) and require semantics of the researcher's subject and the use of other methods in the future.

\section{TABLES AND FIGURES}

\subsection{Figure}

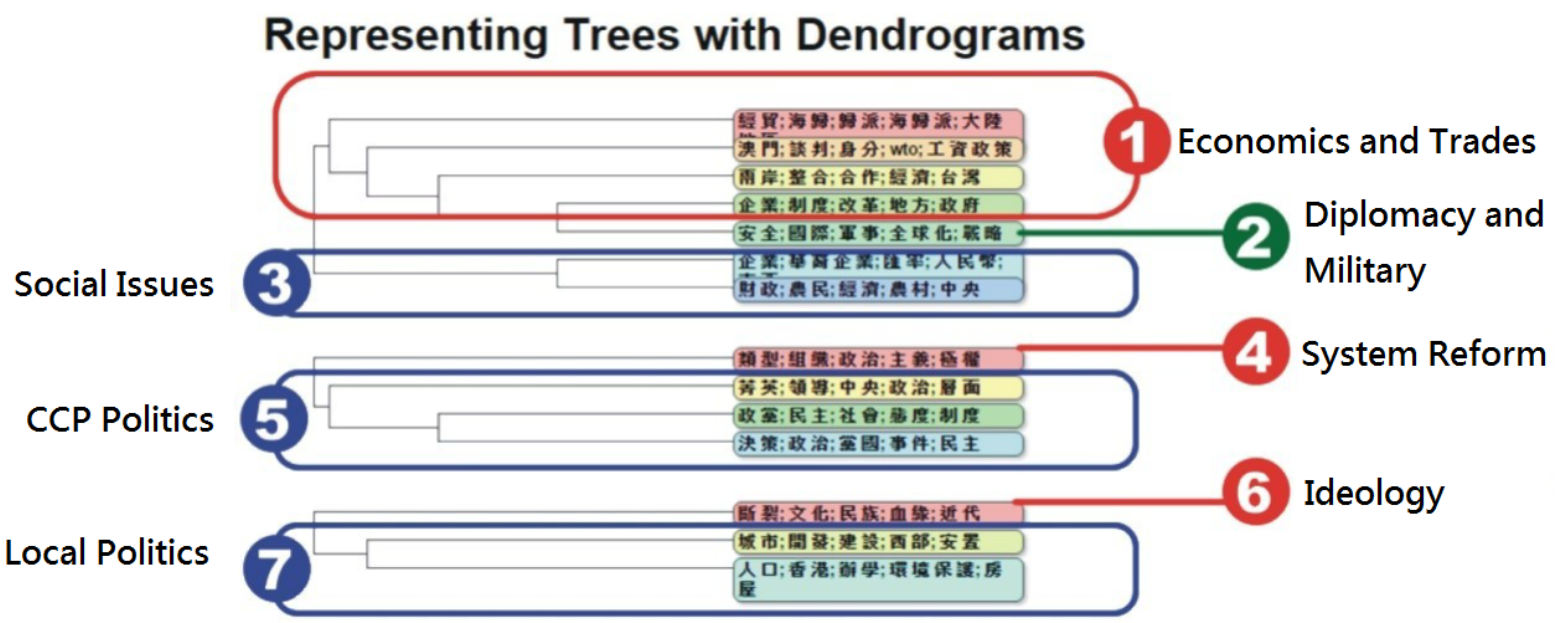

Figure 1: SMD of China Studies in Taiwan 


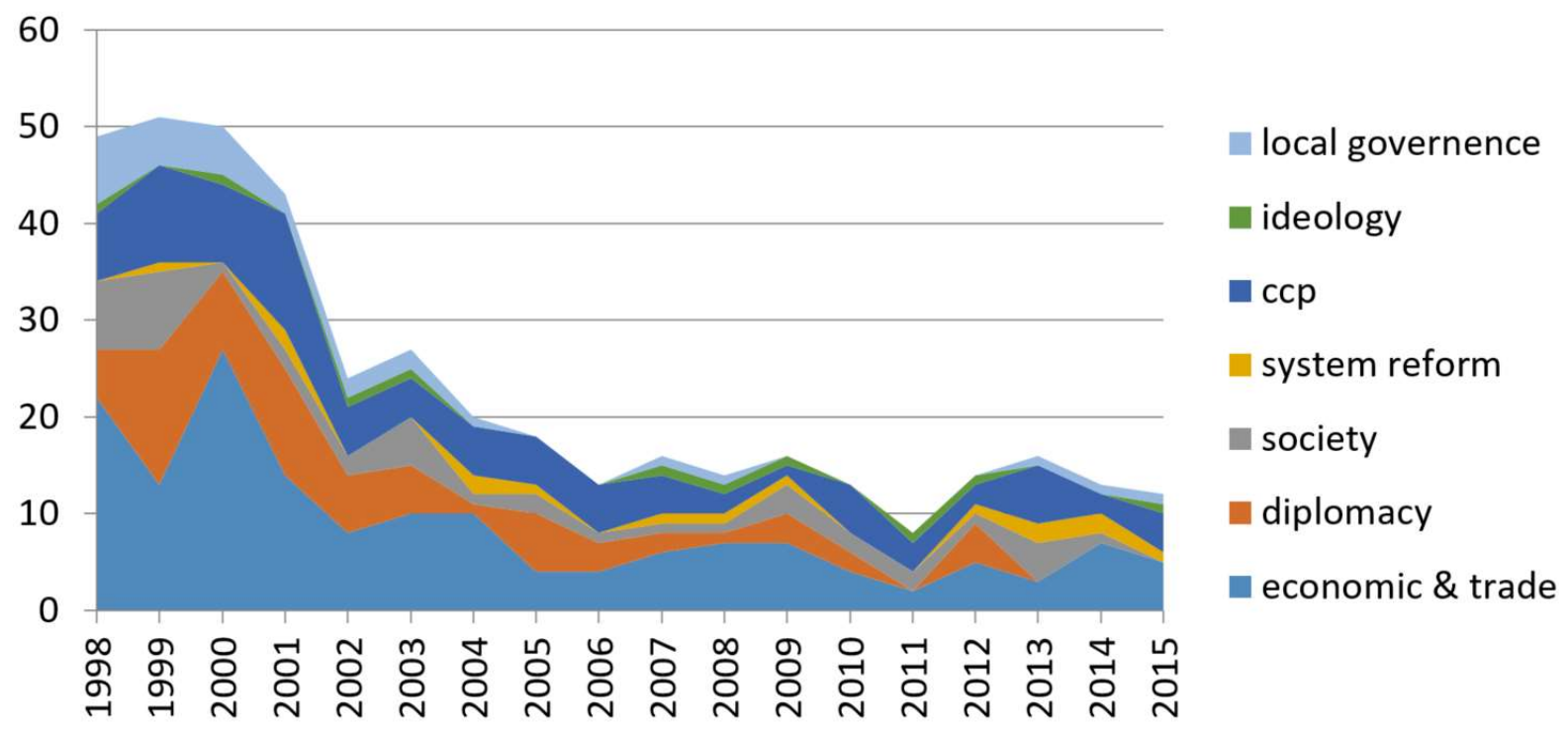

Figure 2: Line Chart of Article Numbers by Year

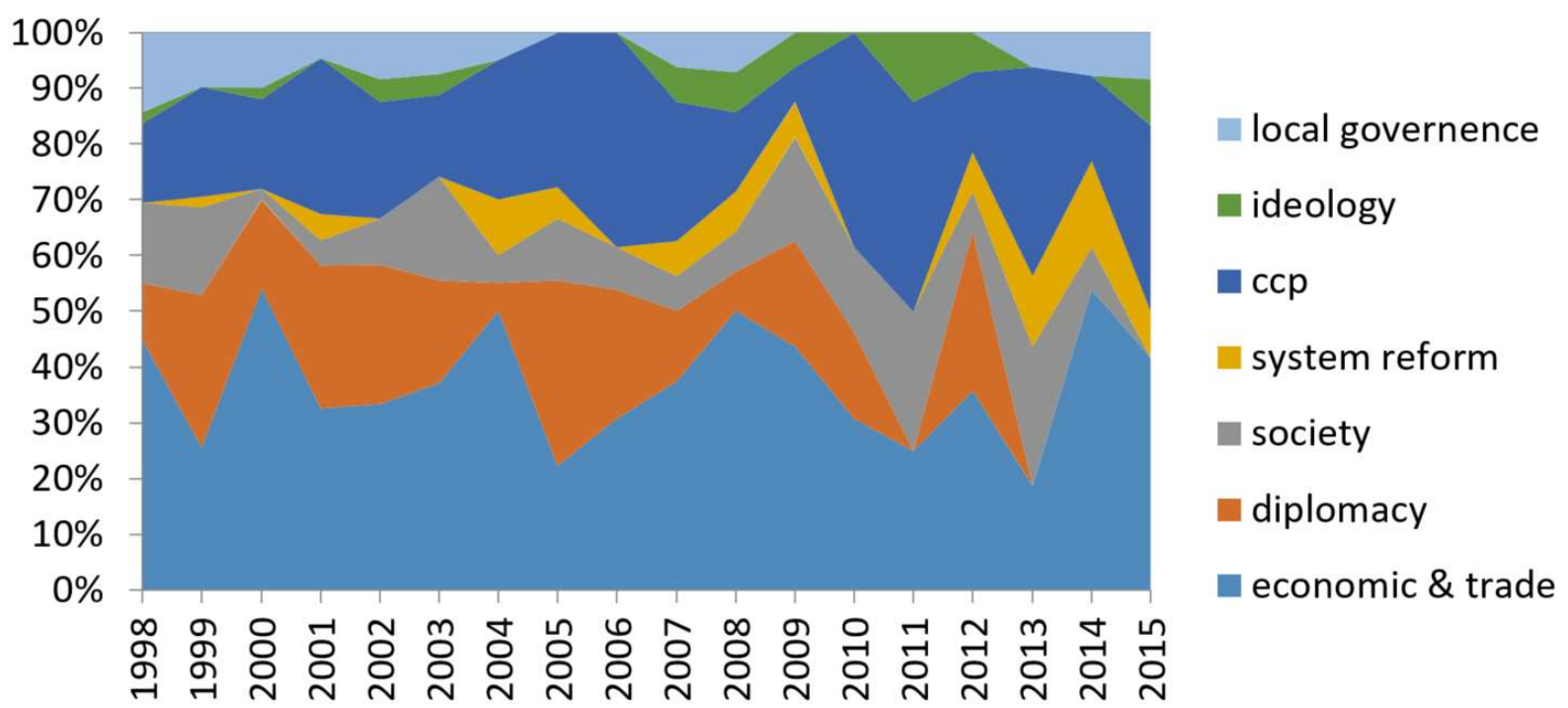

Figure 3: Line Chart of Article Percentages by Year

\subsection{Table}


Table 1: Article Numbers by Year

\begin{tabular}{|l|l|l|l|l|l|l|l|l|}
\hline & $\begin{array}{l}\text { Economic } \\
\& \text { Trade }\end{array}$ & Diplomacy & Society & System Reform & CCP & Ideology & $\begin{array}{l}\text { Local } \\
\text { Governance }\end{array}$ & Total \\
\hline 1998 & 22 & 5 & 7 & 0 & 7 & 1 & 7 & 49 \\
\hline 1999 & 13 & 14 & 8 & 1 & 10 & 0 & 5 & 51 \\
\hline 2000 & 27 & 8 & 1 & 0 & 8 & 1 & 5 & 50 \\
\hline 2001 & 14 & 11 & 2 & 2 & 12 & 0 & 2 & 43 \\
\hline 2002 & 8 & 6 & 2 & 0 & 5 & 1 & 2 & 24 \\
\hline 2003 & 10 & 5 & 5 & 0 & 4 & 1 & 2 & 27 \\
\hline 2004 & 10 & 1 & 1 & 2 & 5 & 0 & 1 & 20 \\
\hline 2005 & 4 & 6 & 2 & 1 & 5 & 0 & 0 & 18 \\
\hline 2006 & 4 & 3 & 1 & 0 & 5 & 0 & 0 & 13 \\
\hline 2007 & 6 & 2 & 1 & 1 & 4 & 1 & 1 & 16 \\
\hline 2008 & 7 & 1 & 1 & 1 & 2 & 1 & 1 & 14 \\
\hline 2009 & 7 & 3 & 3 & 1 & 1 & 1 & 0 & 16 \\
\hline 2010 & 4 & 2 & 2 & 0 & 5 & 0 & 0 & 13 \\
\hline 2011 & 2 & 0 & 2 & 0 & 3 & 1 & 0 & 8 \\
\hline 2012 & 5 & 4 & 1 & 1 & 2 & 1 & 0 & 14 \\
\hline 2013 & 3 & 0 & 4 & 2 & 6 & 0 & 1 & 16 \\
\hline 2014 & 7 & 0 & 1 & 2 & 2 & 0 & 1 & 13 \\
\hline 2015 & 5 & 0 & 0 & 1 & 4 & 1 & 1 & 12 \\
\hline Total & 158 & 71 & 44 & 15 & 90 & 10 & 29 & 417 \\
\hline
\end{tabular}

\section{References}

Zonghe Bao and Yushan Wu. The Theory of Cross-Strait Relations in Argument. Wunan Book Co., Ltd., 2011.

Margeret Hall Benedikt Boecking and Jeff Schneider. Event prediction with learning algorithmsa study of events surrounding the egyptian revolution of 2011 on the basis of micro blog data. Policy \& Internet, 7(2):159-184, 2015.

Rens Vliegenthart Bjorn Burscher and Claes H. De Vreese. Using supervised machine learning to code policy issues: Can classifiers generalize across contexts? The ANNALS of the American Academy of Political and Social Science, 659(1):122-131, 2015.

Michael Finegold, Jessica Otis, Cosma Shalizi, Daniel Shore, Lawrence Wang, and Christopher Warren. Six degrees of francis bacon: a statistical method for reconstructing large historical social networks. Digital Humanities Quarterly, 10(3), 2016.

Justin Grimmer and Brandon M Stewart. Text as data: The promise and pitfalls of automatic content analysis methods for political texts. Political analysis, 21(3):267-297, 2013.

Harold Lasswell. Who gets what, when, how. Whittlesey House, New York-London, 1936.

Grace Muzny, Mark Algee-Hewitt, and Dan Jurafsky. Dialogism in the novel: A computational model of the dialogic nature of narration and quotations. Digital Scholarship in the Humanities, 32(suppl_2):ii31-ii52, 2017.

Margaret E Roberts, Brandon M Stewart, Dustin Tingley, Christopher Lucas, Jetson Leder-Luis, Shana Kushner Gadarian, Bethany Albertson, and David G Rand. Structural topic models for open-ended survey responses. American Journal of Political Science, 58(4):1064-1082, 2014.

De-Sheng Chen Si-Yin Ho and Shu Geng. Research Methods and Achievements in Mainland China. Institute of International Relations, NCCU, 2005.

Donald Sturgeon. Unsupervised identification of text reuse in early chinese literature. Digital Scholarship in the Humanities, page fqx024, 2017. doi: 10.1093/llc/fqx024. URL http://dx.doi.org/10.1093/11c/ fqx024.

Yuen-Hsien Tseng. Generic title labeling for clustered documents. Expert Systems with Applications, 37(3): 2247-2254, 2010.

Yuen-Hsien Tseng, Yu-I Lin, Yi-Yang Lee, Wen-Chi Hung, and Chun-Hsiang Lee. A comparison of methods for detecting hot topics. Scientometrics, 81(1):73-90, 2009.

John Wilkerson and Andreu Casas. Large-scale computerized text analysis in political science: Opportunities and challenges. Annual Review of Political Science, 20:529-544, 2017. 